Research Paper

\title{
Tension Force Downregulates Matrix Metalloprotein- ase Expression and Upregulates the Expression of Their Inhibitors through MAPK Signaling Pathways in MC3T3-El cells
}

\author{
Yoko Karasawa1, Hideki Tanaka2,3, Kumiko Nakai2,3, Natsuko Tanabe3,4, Takayuki Kawato2,3凶, Masao \\ Maeno ${ }^{2,3}$, Noriyoshi Shimizu ${ }^{5,6}$ \\ 1. Nihon University Graduate School of Dentistry, Tokyo, Japan \\ 2. Department of Oral Health Sciences, Nihon University School of Dentistry, Tokyo, Japan \\ 3. Division of Functional Morphology, Dental Research Center, Nihon University School of Dentistry, Tokyo, Japan \\ 4. Department of Biochemistry, Nihon University School of Dentistry, Tokyo, Japan \\ 5. Department of Orthodontics, Nihon University School of Dentistry, Tokyo, Japan \\ 6. Division of Clinical Research, Dental Research Center, Nihon University School of Dentistry, Tokyo, Japan \\ $\square$ Corresponding author: Dr. Takayuki Kawato, Department of Oral Health Sciences, Nihon University School of Dentistry, 1-8-13 Kanda-Surugadai, \\ Chiyoda-ku, Tokyo 101-8310, Japan. Fax: +81-3-3219-8138; E-mail: kawato.takayuki@nihon-u.ac.jp
}

() 2015 Ivyspring International Publisher. Reproduction is permitted for personal, noncommercial use, provided that the article is in whole, unmodified, and properly cited. See http:/ /ivyspring.com/terms for terms and conditions.

Received: 2015.07.16; Accepted: 2015.09.20; Published: 2015.10.22

\begin{abstract}
Objective: Matrix metalloproteinases (MMPs), produced by osteoblasts, catalyze the turnover of extracellular matrix (ECM) molecules in osteoid, and the regulation of MMP activity depends on interactions between MMPs and tissue inhibitors of metalloproteinases (TIMPs). We focused on the degradation process of ECM in osteoid that was exposed to mechanical strain, and conducted an in vitro study using MC3T3-El osteoblastic cells to examine the effects of tension force (TF) on the expression of MMPs and TIMPs, and activation of mitogen-activated protein kinase (MAPK) pathways.

Design: Cells were incubated on flexible-bottomed culture plates and stimulated with or without cyclic TF for 24 hours. The expression of MMPs and TIMPs was examined at mRNA and protein levels by real-time RT-PCR and Western blotting, respectively. The phosphorylation of extracellular signal-regulated kinase (ERK) 1/2, p38 MAPK, and stress-activated protein kinases/c-jun $\mathrm{N}$-terminal kinases (SAPK/JNK) were examined by Western blotting.

Results: TF decreased the expression of MMP-1, $-3,-13$ and phosphorylated ERK1/2. In contrast, TF increased the expression of TIMP-2, -3 and phosphorylated SAPK/JNK. The expression of MMP-2, -14, TIMP-1, -4 and phosphorylated P38 MAPK was unaffected by TF. MMP-1, -3 and -13 expression decreased in cells treated with the ERK inhibitor PD98059 compared with untreated control cells. The JNK inhibitor SP600125 inhibited the TF-induced upregulation of TIMP-2 and -3.

Conclusions: The results suggest that TF suppresses the degradation process that occurs during ECM turnover in osteoid via decreased production of MMP-1, -3 and -13 , and increased production of TIMP-2 and -3 through the MAPK signaling pathways in osteoblasts.
\end{abstract}

Key words: osteoblast, matrix metalloproteinases, tissue inhibitors of metalloproteinases, mechanical strain, mitogen-activated protein kinase

\section{Introduction}

During orthodontic treatment, tooth movement occurs following alveolar bone remodeling induced by the application of therapeutic mechanical strain
$[1,2]$. Osteoblasts perform a central role in bone formation via high alkaline phosphatase activity and the production of collagenous and noncollagenous ex- 
tracellular matrix (ECM) proteins [3-5]. Osteoblasts also produce osteoclast differentiation-related factors including inflammatory cytokines, prostaglandin $\mathrm{E}_{2}$, receptor activator of NF-KB ligand (RANKL), and osteoprotegerin, a natural inhibitor of RANKL [6]. Moreover osteoblasts also produce several kinds of proteinases [7] that are involved in the degradation of ECM proteins in osteoid, which is an important process for osteoclast attachment [8,9]. Thus, osteoblasts regulate not only bone formation, but also bone resorption during the process of bone remodeling. In in vitro experiments using osteoblasts, several previous studies have suggested that mechanical loading, including continual compressive force and cyclic tension force (TF), can affect the expression of osteogenic [10-12] and osteoclast differentiation-related factors [13-15]. These studies were performed to clarify the role of osteoblasts in orthodontic force-induced bone remodeling. However, few studies have investigated the effects of mechanical loading on osteoblast proteinase expression.

Matrix metalloproteinases (MMPs) produced by osteoblasts are active at neutral $\mathrm{pH}$ and can therefore catalyze the turnover of ECM molecules $[7,16]$. The MMP family can be genetically divided into six groups based on their specificity, sequence similarity, and domain organization: collagenases (MMP-1, -8, and -13), gelatinases (MMP-2 and -9), stromelysins (MMP-3, -10, and -11), matrilysins (MMP-7 and -26), membrane-type MMPs (MMP-14 and -17), and other MMPs $[17,18]$. MMP activity depends on interactions between MMPs and tissue inhibitors of metalloproteinases (TIMPs) [18, 19]. Four mammalian TIMPs (TIMP-1, -2, -3, and -4) have been cloned, and their primary structures and functions have been analyzed [20-22]. MMP and TIMP expression is regulated by the mitogen-activated protein kinase (MAPK) pathway in many kinds of cells including fibroblast-like synoviocytes [23], chondrocytes [24], and osteoblasts [25].

We focused on the degradation process of ECM in osteoid that was exposed to mechanical strain, and conducted an in vitro study using MC3T3-E1 osteoblastic cells to examine the effects of tension force (TF) on the expression of MMPs and TIMPs. In addition, the effects of TF on MAPK phosphorylation of extracellular signal-regulated kinase (ERK) 1/2, p38 MAPK, and stress-activated protein kinases/c-jun N-terminal kinases (SAPK/JNK) in MC3T3-E1 cells were evaluated.

\section{Materials and Methods}

\section{Cell Culture}

MC3T3-E1 cells from a mouse calvarial cell line were purchased from Riken Bio Resource Center (Tsukuba, Japan) and used as osteoblasts. Cells were maintained in a-minimal essential medium (a-MEM; Gibco BRL, Rockville, MD, USA), containing 10\% $(\mathrm{v} / \mathrm{v})$ heat-inactivated fetal bovine serum (HyClone Laboratories, Logan, UT, USA) and $1 \%(\mathrm{v} / \mathrm{v})$ penicillin-streptomycin solution (Sigma-Aldrich, St. Louis, $\mathrm{MO}, \mathrm{USA})$, at $37^{\circ} \mathrm{C}$ in a humidified atmosphere of $95 \%$ air and $5 \% \mathrm{CO}_{2}$. The medium was changed every 3 days. Cells were plated on flexible-bottomed six-well culture plates (Flexcell Corp., Hillsborough, NC, USA) at a density of $2 \times 10^{4}$ cells $/ \mathrm{cm}^{2}$.

\section{Application of TF}

Briefly, cyclic TF was applied to MC3T3-E1 cells using a Flexercell Strain Unit (FX-3000, Flexcell Corp.), which mechanically strains the cells. MC3T3-E1 cells were seeded on flexible-bottomed six-well plates with a hydrophilic surface at a density of $2 \times 10^{4}$ cells $/ \mathrm{cm}^{2}$ and then placed onto a vacuum manifold controlled by computer software and a solenoid valve. The system uses a vacuum source to apply a negative pressure causing a downward deformation of the membrane to which the cells are attached. The strain applied over the loading-post region was approximately equal in the radial and circumferential directions [26]. Cells were flexed at 6 cycles/min ( $5 \mathrm{~s}$ strain, $5 \mathrm{~s}$ relaxation) for $0,4,8$ or $12 \%$ TF for 24 hours. TF strength was determined based on previous studies [12, 27-29] using the Flexercell Strain Unit. Controls were prepared in an identical manner and cultured on unstrained flexible-bottomed plates.

\section{Real-time reverse transcription (RT)-polymerase chain reaction (PCR)}

Total RNA was isolated from TF-stimulated or unstimulated cells using NucleoSpin RNA (Takara Bio, Shiga, Japan), and isolated RNA was treated with DNase. The amount of DNase-treated RNA was measured using a NanoDrop 1000 (ND-1000; Thermo Fisher Scientific, Wilmington, DE, USA) and was converted into complementary DNA (cDNA) using an RNA PCR kit (PrimScript; Takara Bio). The cDNA $(0.2 \mu \mathrm{g} / 2 \mu \mathrm{L})$ was subjected to real-time PCR using SYBR Green I dye. Reactions were performed in $25 \mu \mathrm{L}$ SYBR $^{\circledR}$ premixed Ex Taq $^{\mathrm{TM}}$ solution (Takara Bio), containing $20 \mu \mathrm{M}$ sense and anti-sense primers (Table 1). The PCR assays were performed on a Smart Cycler (Cepheid, Sunnyvale, CA, USA) and analyzed using Smart Cycler software. The PCR protocol for MMPs, TIMPs and glyceraldehyde 3-phosphate dehydrogenase (GAPDH), consisted of 30 cycles of $95^{\circ} \mathrm{C}$ for 3 $\mathrm{s}$ and $60^{\circ} \mathrm{C}$ for $20 \mathrm{~s}$. All real-time RT-PCR experiments were performed in triplicate, and the specificity of the PCR products was verified by melt curve analysis. 
The expression levels of MMPs and TIMPs were normalized to that of GAPDH.

\section{SDS-PAGE and Western blotting}

Cells were lysed in extraction buffer containing $0.05 \%$ Triton X-100, $10 \mathrm{mM} \quad \beta$-mercaptoethanol, $0.5 \mathrm{mM}$ phenylmethylsulfonyl fluoride, $0.5 \mathrm{mM}$ ethylenediaminetetraacetic acid, and $25 \mathrm{mM}$ Tris- $\mathrm{HCl}$ ( $\mathrm{pH}$ 7.4). Cell membranes were disrupted by sonication, and the samples were clarified by centrifugation. Supernatants containing $20 \mu \mathrm{g}$ intracellular protein were dissolved in $10 \mu \mathrm{L}$ sample buffer containing $1 \%$ sodium dodecyl sulfate (SDS), $2 \mathrm{M}$ urea, $15 \mathrm{mg} / \mathrm{mL}$ dithiothreitol, and bromophenol blue and then heated at $95^{\circ} \mathrm{C}$ for $5 \mathrm{~min}$ prior to loading. Proteins were resolved by 4-20\% SDS-polyacrylamide gel electrophoresis (SDS-PAGE) using a discontinuous Tris-glycine buffer system [30], transferred to a polyvinylidene fluoride membrane using a semidry transfer apparatus, and probed with the indicated antibodies. Polyclonal or monoclonal IgG primary antibodies, including rabbit anti-MMP-2, -MMP-13, -TIMP-1, goat anti-MMP-1, -MMP-3, -TIMP-2, -TIMP-3, -TIMP-4, mouse anti- $\beta$-tubulin (Santa Cruz Biotechnology, Santa Cruz, CA, USA), rabbit anti-MMP-14 (Assaybiotech, Sunnyvale, CA, USA), rabbit anti-ERK1/2, -phospho-ERK1/2, -p38 MAPK, -phospho-p38 MAPK, -SAPK/JNK, and -phospho-SAPK/JNK (Cell Signaling Technology, Danvers, MA, USA) were used with the appropriate biotin-conjugated donkey anti-goat IgG (Merck Millipore, Darmstadt, Germany), goat anti-rabbit IgG (Zymed, San Francisco, CA, USA), or goat anti-mouse IgG (Abcam plc, Cambridge, UK) secondary antibodies. The membranes were labeled with streptavidin-horseradish peroxidase (streptavidin-HRP) and visualized using a commercial chemiluminescence kit (Amersham Life Sciences, Little Chalfont, Buckinghamshire, UK). To re-probe with different antibodies, the membrane was stripped with Restore PLUS Western blot stripping buffer (Thermo Fisher Scien- tific) at room temperature for $15 \mathrm{~min}$.

\section{Treatment of MC3T3-El cells with MAPK in-} hibitors

To investigate the role of ERK1/2 and SAPK/JNK signaling on MMP and TIMP expression in MC3T3-E1 cells, the cells were treated with the ERK inhibitor PD98059 $(10 \mu \mathrm{M}$; Merck Millipore) or the JNK inhibitor SP600125 (1 $\mu \mathrm{M}$; Sigma Aldrich). In the JNK inhibitor experiment, cells were treated with SP600125 for 1 hour prior to TF loading. The concentration of each inhibitor used was based on the manufacturer's instructions and a previous report [25]. Cell status was examined using inverted light microscopy, and mRNA levels were then determined by real-time RT-PCR.

\section{Statistical analysis}

Each value is reported as the mean \pm standard deviation (SD). Significant differences were determined using a student's t-test or one-way analysis of variance (ANOVA) followed by Tukey's multiple comparison test. Differences with a $p$ value $<0.05$ were considered statistically significant.

\section{Results}

\section{Effect of TF on MMP and TIMP mRNA ex- pression}

MC3T3-E1 cells were stimulated with 0 (control), 4,8 , or $12 \%$ TF for 24 hours, and the mRNA expression of MMPs (MMP-1, -2, $-3,-13$ and -14) and TIMPs (TIMP-1, -2, -3 and -4) was determined. The expression of MMP-1, -3 and -13 was significantly decreased in 4,8 and/or $12 \%$ TF compared with $0 \%$ TF (Figure $1 \mathrm{~A}, \mathrm{C}$ and D). There was no marked difference in the amount of MMP-1 and - 3 inhibition between different TF magnitudes (percentage); however, MMP-13 expression gradually decreased with increasing TF magnitude. TF did not affect the expression of MMP-2 or -14 (Figure 1B and E).

Table 1. PCR primers used in the experiments.

\begin{tabular}{lll}
\hline Target & Forward primer & Reverse primer \\
\hline MMP-1 & 5'-CACATTGATGCTGCTGTTGTGA-3' & 5'-TCTGCTGTTAATCTGGGATAACCTG-3' \\
MMP-2 & 5'-GATAACCTGGATGCCGTCGTG-3' & 5'-GGTGTGCAGCGATGAAGATGATA-3' \\
MMP-3 & 5'-CTCATGCCTATGCACCTGGAC-3' & 5'-TCATGAGCAGCAACCAGGAA-3' \\
MMP-13 & 5'-TCCCTGGAATTGGCAACAAAG-3' & 5'-GCATGACTCTCACAATGCGATTAC-3' \\
MMP-14 & 5'-GCAGTGGACAGCGAGTACCCTA-3' & 5'-AGTCCCGCAGAGCTGACTTG-3' \\
TIMP-1 & 5'-CTATAGTGCTGGCTGTGGGGTGTG-3' & 5'-TTCCGTGGCAGGCAAGCAAAGT-3' \\
TIMP-2 & 5'-GGCCTCCCTCCCTTACTCC-3' & 5'-GACTTCATATTCCAGCACGCACAT-3' \\
TIMP-3 & 5'-CTAAGTCGGCTGTTTGGGTTGA-3' & 5'-CAGCACAGCTGGCTTGCTAGA-3' \\
TIMP-4 & 5'-GCCTGAATCATCACTACCACCAGA-3' & 5'-TGAGATGGTACATGGCACTGCATA-3' \\
GAPDH & 5'-AAATGGTGAAGGTCGGTGTG-3' & 5'-TGAAGGGGTCGTTGATGG-3'
\end{tabular}


A

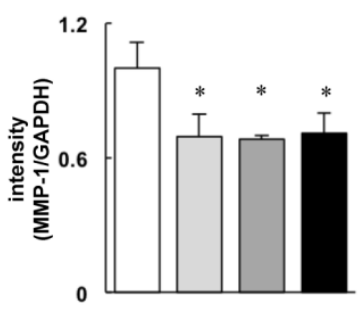

B

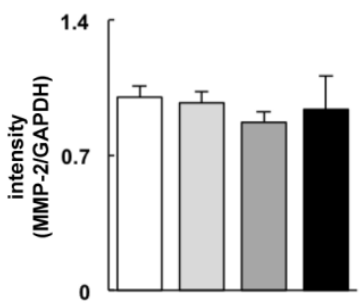

E

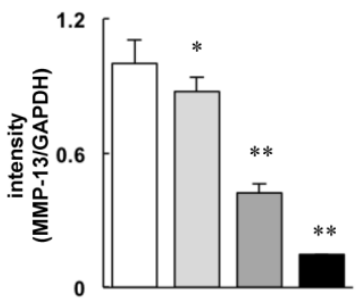

D

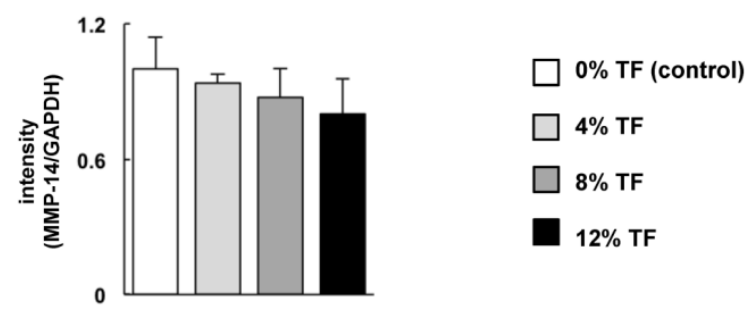

Fig. 1. Effect of TF on MMP mRNA expression. MC3T3-El cells were subjected to 0 (control), 4, 8, or 12\% TF for 24 hours, and the expression of MMP-1 (A), -2 (B), $-3(C),-13$ (D) and -14 (E) was determined using real-time RT-PCR. Gene expression is described as the fold change relative to the control group. Each bar indicates the mean \pm SD from three independent experiments. ${ }^{*} p<0.01,{ }^{*} p<0.05$ (vs. control).

A
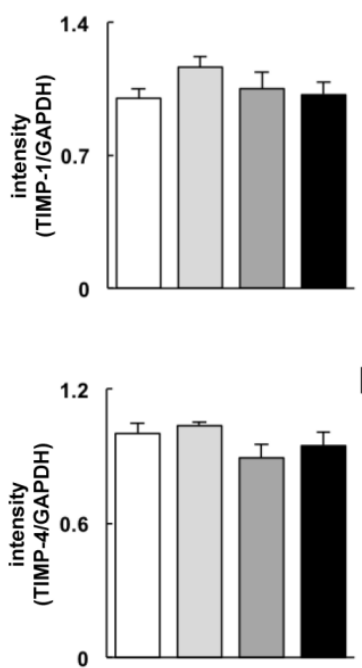

B
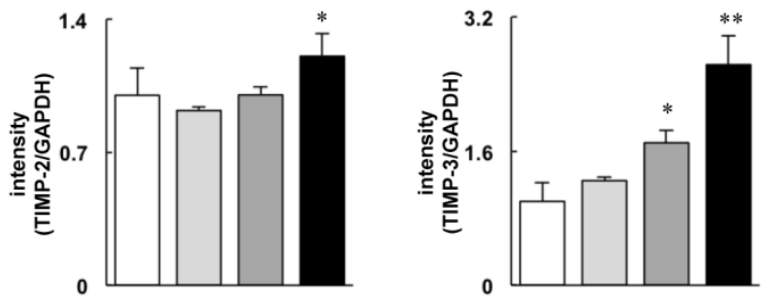

C

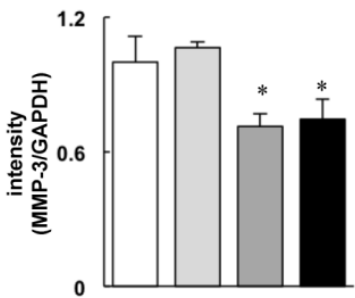

D

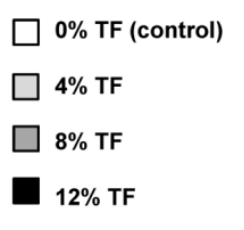

Fig. 2. Effect of TF on TIMP mRNA expression. MC3T3-E1 cells were subjected to $0 \%$ (control), 4,8 , or $12 \%$ TF for 24 hours, and the expression of TIMP-1 (A), -2 (B), $-3(C)$ and -4 (D) was determined using real-time RT-PCR. Gene expression is described as the fold change relative to the control group. Each bar indicates the mean \pm SD from three independent experiments. ** $p<0.01,{ }^{*} p<0.05$ (vs. control).

The effect of TF on TIMP expression was opposite to that on MMP expression. The expression of TIMP-3 was increased significantly at $8 \%$ TF and increased further at $12 \%$, and the expression of TIMP-2 was increased at $12 \%$ TF compared with $0 \%$ TF (Figure $2 \mathrm{~B}$ and $\mathrm{C})$. TF did not affect the mRNA expression of TIMP-1 or -4 (Figure 2A and D).

\section{Effect of TF on MMP and TIMP protein ex- pression}

The cells were stimulated with $12 \% \mathrm{TF}$, which strongly inhibited MMP-13 expression and promoted TIMP-3 expression at the mRNA level as shown in
Figure 1D and 2C, and the protein levels of MMPs (MMP-1, -2, -3, -13 and -14) and TIMPs (TIMP-1, -2, -3 and -4$)$ were determined. After $12 \% \mathrm{TF}$ stimulation, the expression levels of MMP-1, -3 and -13 were significantly decreased compared with $0 \%$ TF (Figure $3 \mathrm{~A}, \mathrm{C}$ and D), while MMP-2 and -14 were unaffected by $12 \%$ TF (Figure 3B and E). The expression of TIMP-2 and -3 was significantly increased after $12 \%$ TF compared with $0 \% \mathrm{TF}$, but TF did not affect TIMP-1 or -4 expression (Figure $4 A-D$ ). Thus, the effect of $12 \%$ TF on MMP and TIMP mRNA expression agreed with the protein expression data. 


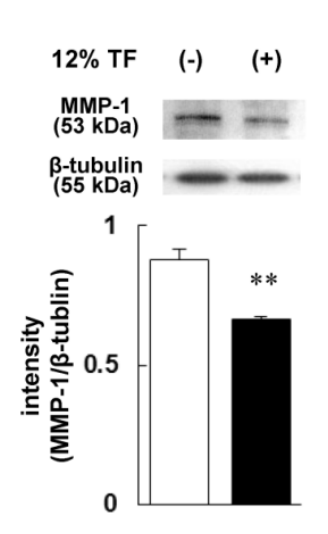

A $\quad 12 \%$ TF $(-) \quad(+) \quad$ B

C
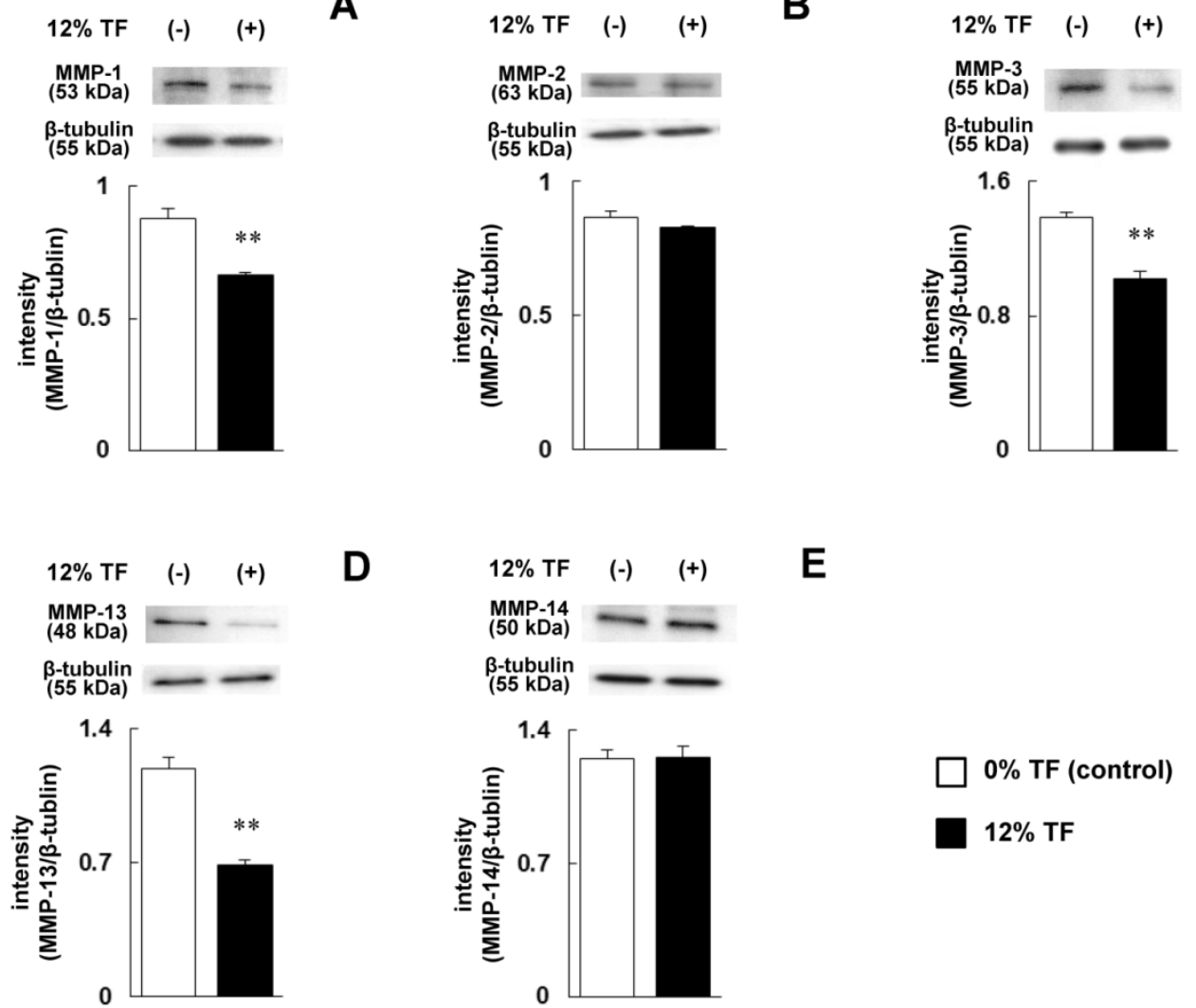

Fig. 3. Effect of TF on MMP protein expression. MC3T3-El cells were subjected to $0 \%$ (control) or $12 \%$ TF for 24 hours, and the expression of MMP-1 (A), -2 (B), $-3(C),-13(D)$ and -14 (E) was examined by Western blot analysis. Each bar indicates the mean \pm SD from three independent experiments. ${ }^{* *} p<0.01$ (vs. control).

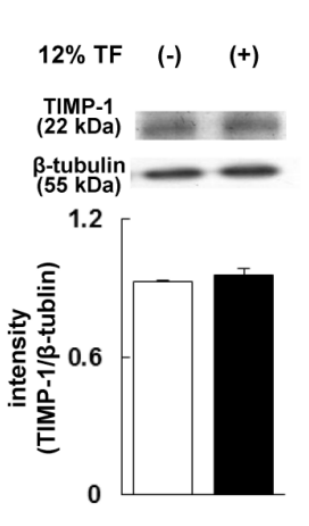

A

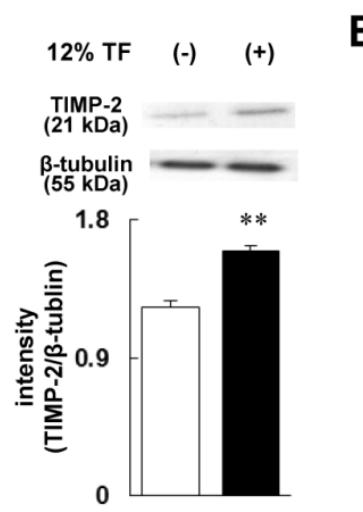

B

C

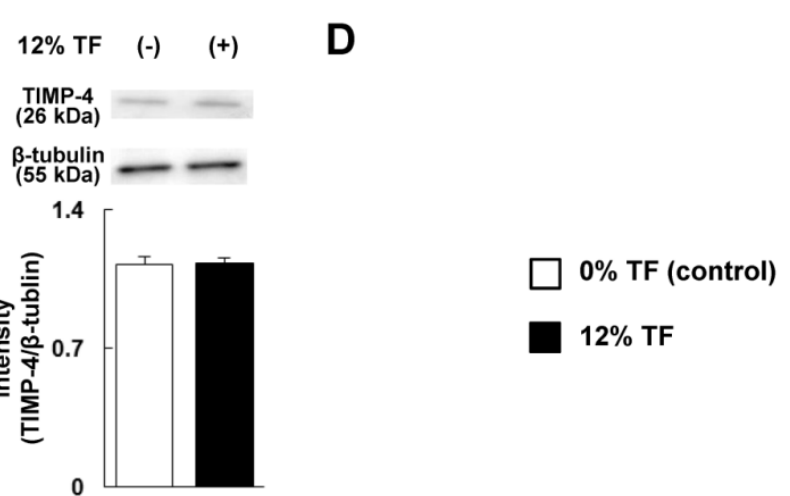

Fig. 4. Effect of TF on TIMP protein expression. MC3T3-El cells were subjected to $0 \%$ (control) or $12 \%$ TF for 24 hours, and the expression of TIMP-1 (A), -2 (B), $-3(C)$, and $-4(D)$ was examined by Western blot analysis. Each bar indicates the mean \pm SD from three independent experiments. ** $p<0.01$ (vs. control). 
Effect of TF on the phosphorylation of ERK1/2, p38 MAPK, or SAPK/JNK

The phosphorylation of ERK1/2, p38 MAPK, and SAPK/JNK in cells after stimulating with TF for 12 and 24 hours was examined by Western blotting. Twelve hours after 12\% TF, ERK1/2 phosphorylation was decreased (Figure 5A), and SAPK/JNK phosphorylation was increased (Figure 5C), and these affects were enhanced after 24 hours. TF did not affect the phosphorylation of p38 MAPK (Figure 5B).

\section{Effect of ERK1/2 and SAPK/JNK phosphoryla- tion inhibitors on MMP and TIMP expression}

The decrease in ERK1/2 phosphorylation and the increase in SAPK/JNK phosphorylation may be involved in the downregulation of MMP-1, -3 , and -13 and the upregulation of TIMP-2 and -3 by TF. To examine these effects more closely, MC3T3-E1 cells were treated with an ERK1/2 specific inhibitor to mimic the TF-induced decrease in ERK1/2 phosphorylation, and the effects of this inhibitor on MMP expression were determined by real-time RT-PCR. Compared with untreated cells, MMP-1, -3 and -13 expression was decreased in cells treated with PD98059 (Figure 6A-C); however, PD98059 did not affect the expression of TIMP-2 or -3 (Figure 6D and E). Next, the effects of the JNK inhibitor SP600125 were determined in cells that were exposed to TF. SP600125 completely inhibited the TF-induced upregulation of TIMP-2 and -3 (Figure 7D and E) but had no effect on the downregulation of MMP-1, -3 or -13 (Figure 7A-C).
A

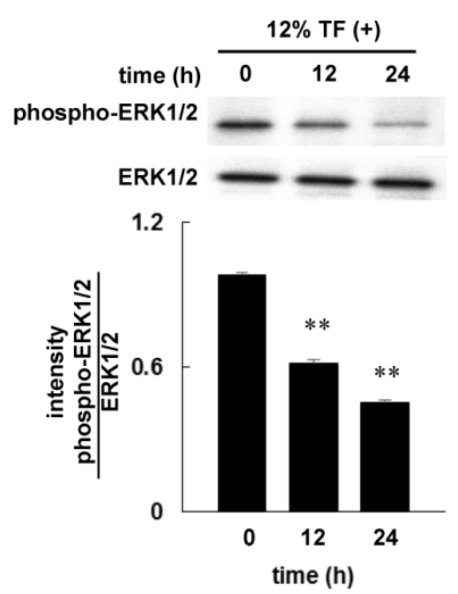

B

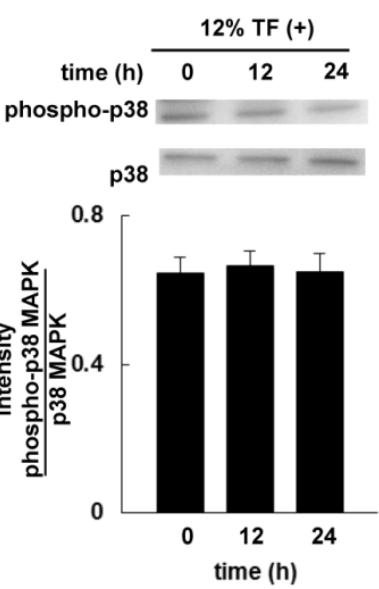

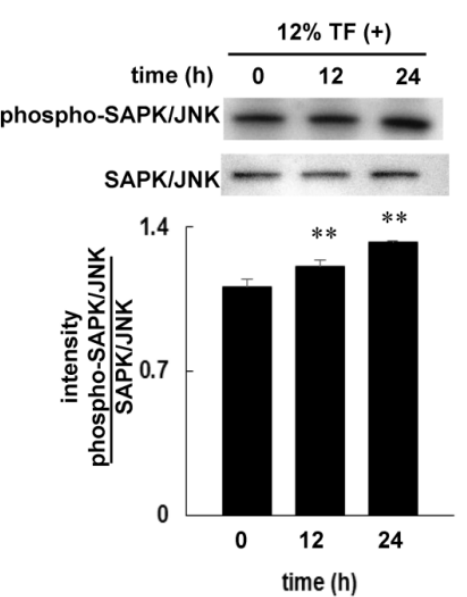

Fig. 5. Effect of TF on the phosphorylation of ERK1/2, p38 MAPK, and SAPK/JNK. MC3T3-E1 cells were subjected to $12 \%$ TF for up to 24 hours, and the phosphorylation of ERK1/2 (A), p38 MAPK (B), and SAPK/JNK (C) was examined by Western blot at 0 (prior to TF application), 12, and 24 hours of culture. Each bar indicates the mean \pm SD from three independent experiments. ${ }^{* *} p<0.01$ (vs. control).

A

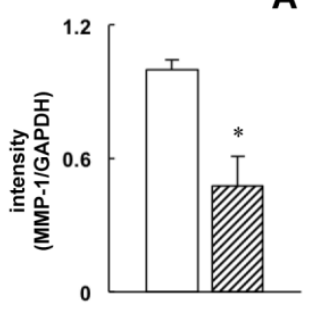

D

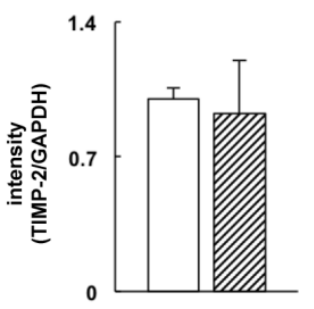

B

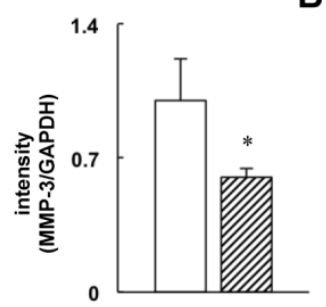

E

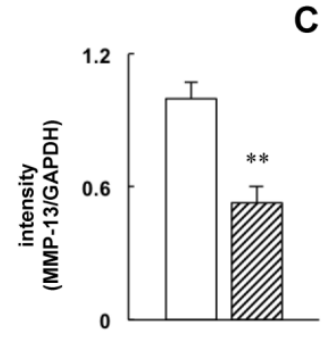

$0 \mu \mathrm{M}$ PD98059 (control)

\ $10 \mu \mathrm{M}$ PD98059

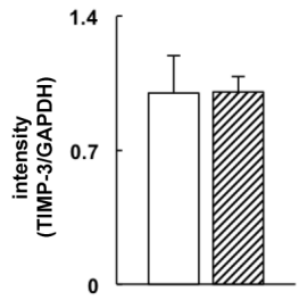

Fig. 6. Effect of PD98059 on MMP-1, -3 and -13 and TIMP-2 and -3 mRNA expression. MC3T3-E1 cells were treated with or without $10 \mu M$ PD98059 (ERK inhibitor) for 24 hours, and the expression of MMP-1 (A), $-3(B)$, and -13 (C) and TIMP-2 (D) and -3 (E) was determined by real-time RT-PCR. The expression level is presented as the fold change relative to the control group. Each bar indicates the mean \pm SD from three independent experiments. ${ }^{* *} p<0.01$, ${ }^{*} p<0.05$ (vs. control). 
A
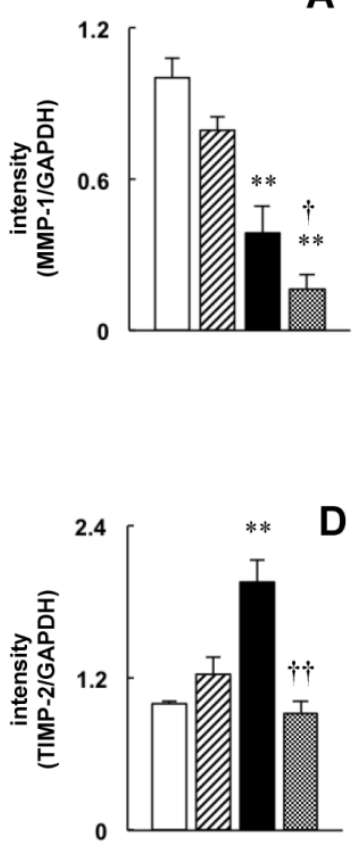

B
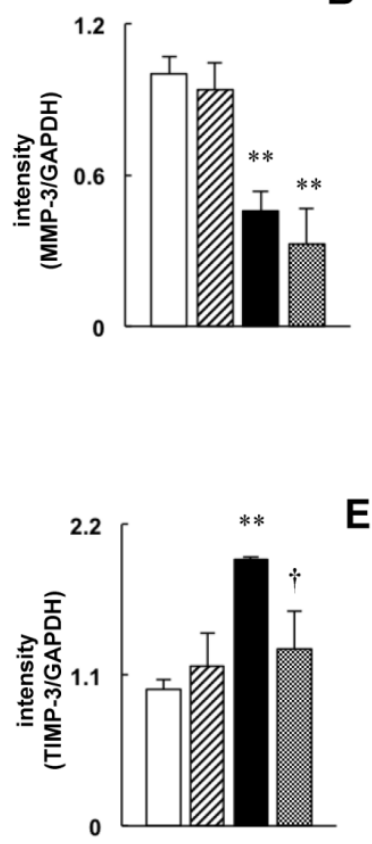

C

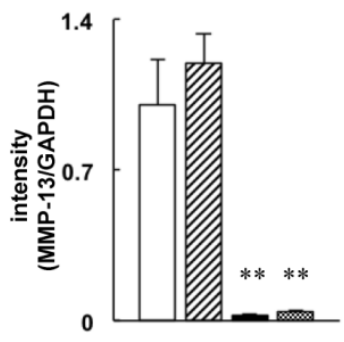

Fig. 7. Effect of SP600125 on TF-induced downregulation of MMP-1, -3 and -13 expression and upregulation of TIMP-2 and -3 expression. MC 3 T3-E1 cells were pretreated with $10 \mu \mathrm{M} \mathrm{SP} 600125$ (JNK inhibitor) and then subjected to 0 (control) or $12 \%$ TF for $24 \mathrm{~h}$. The expression of MMP-1 (A), -3 (B), and -13 (C) and TIMP-2 (D) and $-3(E)$ was determined by real-time PCR. The expression level is presented as the fold change relative to the control group. Each bar indicates the mean \pm SD from three independent experiments. ${ }^{* *} p<0.01$ (TF vs. control), $t p<0.05$ (TF vs. TF plus $1 \mu$ M SP600125)

\section{Discussion}

Certain amounts of mechanical strain have been indicated to enhance the osteogenic functions of osteoblasts; however, excessive mechanical strain can suppress function. Jacobs et al. [28] reported that 5\% $\mathrm{TF}$ induced an increase in type I collagen expression and alkaline phosphatase activity, while $10 \%$ TF decreased the expression and activity in human osteoblasts. Fushiki et al. [29] found that calcified nodule formation was reduced when $18 \% \mathrm{TF}$ was applied to rat calvarial progenitor cells. Kariya et al. [12] reported that $6 \%$ and $18 \%$ TF induced the expression of Runx2 and Osterix in MC3T3-E1 cells, both of which are osteoblast differentiation-related transcription factors. They also reported that TF-induced upregulation of Runx2 and Osterix expression was significantly higher after $6 \% \mathrm{TF}$ compared with $18 \% \mathrm{TF}$, and that $6 \% \mathrm{TF}$ also increased alkaline phosphatase activity, ECM protein expression, and calcium content of calcified nodules. Based on these previous studies [12, 28, 29], we hypothesized that when TF enhances the osteogenic function of osteoblasts, osteoblasts' proteolytic processes may be suppressed, which overall may lead to bone production rather than bone resorption. In this study, the application of 4,8 or $12 \%$ TF to MC3T3-E1 cells resulted in decreased expression of MMP-1, -3 and -13 and increased expression of TIMP-2 and -3. MMP-1 and -13 function mainly as collagenases and can cleave undenatured type I col- lagen, the most abundant protein present in the osteoid layer, into characteristic 3:1 length fragments. In contrast, MMP-3 functions mainly as stromelysin-1 and degrades proteoglycan, gelatin, and other non-collagenous proteins [7, 18]. TIMP-2 and -3 inhibit these proteases; TIMP-2 binds to multiple MMPs, and TIMP-3 binds to the ECM proteins [18, 19]. Thus, the results from the present study indicate that, as expected, proteolytic processing is totally inhibited when osteoblasts are subjected to cyclic TF.

MAPK pathways have been associated with several types of mechanical strain [31-36]; however, the effect of mechanical strain on the activation of MAPK pathways is still not known entirely. In the present study, TF was found to induce different effects on ERK1/2, p38 MAPK, and SAPK/JNK phosphorylation. TF inhibited the phosphorylation of ERK1/2, had a stimulatory effect on SAPK/JNK phosphorylation, and had no effect on p38 MAPK phosphorylation. The majority of previous studies have indicated that compressive force [31], TF [32-34], centrifugal force [35] and ultrasound stimulation [36] induce the phosphorylation of ERK1/2, p38 MAPK, and SAPK/JNK. In contrast, Jackson et al. [37] reported that simultaneously treating MC3T3-E1 cells with strain and fluid shear stress for 1 hour per day for 5-30 days inhibited ERK1/2 phosphorylation but had no effect on p38 MAPK phosphorylation. Moreover, Matsuda et al. [38] reported that a single application of TF for 15-60 minutes induced the phosphor- 
ylation of SAPK/JNK, but not ERK1/2 or p38 MAPK, in human periodontal ligament cells. Therefore the findings by Jackson et al. [37] and Matsuda et al. [38] partially agree with the results of the present study.

Several studies have suggested that the decrease in ERK1/2 phosphorylation may be involved in downregulation of MMP and upregulation of TIMP expression [39-42]. Therefore, the suppressive effect of TF on MMP-1, 3 and -13 expression and the stimulatory effect of TF on TIMP-2 and -3 expression was hypothesized to be due to decreased ERK1/2 phosphorylation. Treatment with PD98059, a specific inhibitor of ERK1/2, also resulted in decreased MMP expression, confirming that $\mathrm{TF}$ acts through this pathway to decrease MMP levels; however, no changes were found in TIMP levels, suggesting a different pathway is involved. In the studies described above [39, 41], the decreased phosphorylation of ERK1/2 and increased phosphorylation of p38 MAPK were induced simultaneously by histone deacetylase inhibitors [39] or anti-cancer agents [41]. In contrast, in the present study, decreased ERK1/2 and increased SAPK/JNK phosphorylation were induced by TF, and no effect was found on p38 MAPK phosphorylation. Therefore, the role of SAPK/JNK was next examined using SP600125, a specific inhibitor of SAPK/JNK. SP600125 completely inhibited the TF-induced upregulation of TIMP-2 and -3 but had no effect on the TF-induced downregulation of MMP-1, -3 and -13 . These results indicate that the simultaneous alteration of ERK1/2 and SAPK/JNK phosphorylation results in downregulation and upregulation of MMPs and TIMPs, respectively.

Two previous studies conducted by Yang et al. [34] and $\mathrm{Li}$ et al. [43] reported that TF increased MMP-13 mRNA and protein expression. Yang et al. [34] reported that when $8 \% \mathrm{TF}$ was applied to MC3T3-E1 cells for up to 4 hours, MMP-13 mRNA and protein expression levels increased after 30 minutes and plateaued after 1 hour. The results from the present study and that of Yang et al. [34] indicate a difference between short-term (30 $\mathrm{min}$ ) and long-term (24 hours) TF treatment in osteoblasts, in that short-term treatment increases, and long-term treatment decreases MMP-13 expression. In contrast, Li et al. [43] reported that the effect of TF on MMP-13 expression was dependent upon TF magnitude in MC3T3-E1 cells exposed to 6, 12, and 18\% TF for 24 hours. The reason for the differing results regarding TF effect on MMP-13 between the present study and that by Li et al. [43] is unknown. However, previous studies have suggested that serum concentration may affect the expression of multiple genes, including MMPs, in cultured cells [44]. In this study, culture medium containing 10\% FBS was used for cell growth, maintenance, and stimulation, while Li et al. [43] reduced the concentration of FBS from $10 \%$ to $1 \%$ during TF treatment. This may be a minor difference; however it is possible that different FBS concentrations may affect MMP-13 expression.

Several studies have reported that TF induces downregulation of MMP-1, -3, and -13, and upregulation of TIMP-2 expression in synovial cells $[23,45]$ and tenocytes [46, 47]. In the present study, the TF-induced downregulation of collagenase (MMP-1, and -13) and stromelysin (MMP-3) expression and the upregulation of their natural inhibitors (TIMP-2 and -3) were confirmed in osteoblasts. The removal of collagen and non-collagenous protein from the bone surface is important for osteoclast attachment $[8,9]$. Tang et al. [48] also previously reported that TF induced a decrease in the expression of RANKL, an essential cytokine for osteoclast differentiation, and an increase in the expression of osteoprotegerin, a decoy receptor of RANKL [6] in MC3T3-E1 cells. Both the present results and those of Tang et al. [48] suggest that TF not only suppresses the differentiation of osteoclasts but also their attachment to the TF-exposed bone surface.

In conclusion, the results suggest that TF suppresses the degradation process that occurs during ECM turnover in osteoid via decreasing MMP-1, -3 and -13 expression and increasing TIMP-2 and -3 expression through the MAPK signaling pathway in osteoblasts.

\section{Acknowledgments}

This study was supported by the following: the Promotion and Mutual Aid Corporation for Private Schools of Japan; a grant from the Dental Research Center, Nihon University School of Dentistry; the Sato fund and the Uemura fund, Nihon University School of Dentistry; and the Nihon University Multidisciplinary Research Grant for 2014-2015.

\section{Authors' contributions}

Yoko Karasawa: conceptualized and designed the study and experiments, performed the experiments, analyzed and interpreted the data, and wrote the manuscript.

Hideki Tanaka: designed the experiments, interpreted the data, provided reagents and materials, and reviewed the manuscript.

Kumiko Nakai: designed the experiment, analyzed the data, and provided materials and reagents.

Natsuko Tanabe: interpreted the data and reviewed the manuscript.

Takayuki Kawato: conceptualized and designed the study and experiments, analyzed and interpreted the data, and wrote the manuscript. 
Masao Maeno: Conceptualized and designed the study, provided reagents, materials and equipment, interpreted the data, and reviewed the manuscript.

Noriyoshi Shimizu. Conceptualized and designed the study, provided equipment, interpreted the data, and reviewed the manuscript.

\section{Abbreviations}

MMP: matrix metalloproteinase; ECM: extracellular matrix; TIMP: tissue inhibitors of metalloproteinase; TF: tension force; MAPK: mitogen-activated protein kinase; ERK: extracellular signal-regulated kinase; SAPK/JNK: stress-activated protein kinases/c-jun N-terminal kinases; RANKL: receptor activator of NF-KB ligand.

\section{Competing Interests}

The authors have declared that no competing interest exists.

\section{References}

1. Roberts WE, Goodwin WC, Heiner SR. Cellular response to orthodontic force. Dent Clin North Am. 1981; 25(1): 3-17.

2. Krishnan V, Davidovitch Z. Cellular, molecular, and tissue-level reactions to orthodontic force. Am J Orthod Dentofacial Orthop. 2006; 129(4): 469.e1-32.

3. Anderson HC. Mechanism of mineral formation in bone. Lab Invest. 1989; 60(3): 320-30.

4. Robey PG, Fedarko NS, Hefferan TE, et al. Structure and molecular regulation of bone matrix proteins. J Bone Miner Res. 1993; 8 (Suppl 2): S483-7.

5. Mundlos S and Olsen BR. Heritable diseases of the skeleton. Part II: Molecular insights into skeletal development-matrix components and their homeostasis. FASEB J. 1997; 11(4): 227-33.

6. Maeno M, Tanaka H, Zhang F, et al. Direct and indirect effects of IL-17A on RANKL-induced osteoclastogenesis. J Hard Tissue Biol. 2013; 22(3): 287-92.

7. Paiva KB, Granjeiro JM. Bone tissue remodeling and development: focus on matrix metalloproteinase functions. Arch Biochem Biophys. 2014; 561: 74-87.

8. Holliday LS, Welgus HG, Fliszar CI, et al. Initiation of osteoclast bone resorption by interstitial collagenase. J Biol Chem. 1997; 272(35): 22053-8.

9. Zhao $\mathrm{W}$, Byrne $\mathrm{MH}$, Boyce BF, et al. Bone resorption induced by parathyroid hormone is strikingly diminished in collagenase-resistant mutant mice. J Clin Invest. 1999; 103(4): 517-24.

10. Mitsui N, Suzuki N, Maeno M, et al. Optimal compressive force induces bone formation via increasing bone sialoprotein and prostaglandin $\mathrm{E}_{2}$ production appropriately. Life Sci. 2005; 77(25): 3168-82

11. Shionome C, Kawato T, Tanabe N, et al. Compressive force induces the expression of bone remodeling-related proteins via interleukin-11 production in MC3T3-E1 cells. J Hard Tissue Biol. 2012; 21(1): 65-74

12. Kariya T, Tanabe N, Shionome C, et al. Tension force-induced ATP promotes osteogenesis through P2X7 receptor in osteoblasts. J Cell Biochem. 2015; 116(1): 12-21.

13. Koyama Y, Mitsui N, Suzuki N, et al. Effect of compressive force on the expression of inflammatory cytokines and their receptors in osteoblastic Saos-2 cells. Arch Oral Biol. 2008; 53(5): 488-96.

14. Sanuki R, Shionome C, Kuwabara A, et al. Compressive force induces osteoclast differentiation via prostaglandin $\mathrm{E}_{2}$ production in MC3T3-E1 cells. Connect Tissue Res. 2010; 51(2): 150-8.

15. Zhang F, Wang CL, Koyama Y, et al. Compressive force stimulates the gene expression of IL-17s and their receptors in MC3T3-E1 cells. Connect Tissue Res. 2010; 51(5): 359-69.

16. Malemud CJ. Matrix metalloproteinases (MMPs) in health and disease: an overview. Front Biosci. 2006; 11: 1696-701.

17. Egeblad M, Werb Z. New functions for the matrix metalloproteinases in cancer progression. Nat Rev Cancer. 2002; 2(3): 161-74.

18. Nagase H, Visse R, Murphy G. Structure and function of matrix metalloproteinases and TIMPs. Cardiovasc Res. 2006; 69(3): 562-73.

19. Visse R, Nagase H. Matrix metalloproteinases and tissue inhibitors of metalloproteinases: structure, function, and biochemistry. Circ Res. 2003; 92(8): 827-39.

20. Olson MW, Gervasi DC, Mobashery S, et al. Kinetic analysis of the binding of human matrix metalloproteinase-2 and -9 to tissue inhibitor of metalloproteinase (TIMP)-1 and TIMP-2. J Biol Chem. 1997; 272(47): 29975-83.

21. Yu WH, Yu S, Meng Q, et al. TIMP-3 binds to sulfated glycosaminoglycans of the extracellular matrix. J Biol Chem. 2000; 275(40): 31226-32.
22. Huang W, Li WQ, Dehnade F, et al. Tissue inhibitor of metalloproteinases-4 (TIMP-4) gene expression is increased in human osteoarthritic femoral head cartilage. J Cell Biochem. 2002; 85(2): 295-303

23. Wang P, Yang L, You X, et al. Mechanical stretch regulates the expression of matrix metalloproteinase in rheumatoid arthritis fibroblast-like synoviocytes. Connect Tissue Res. 2009; 50(2): 98-109.

24. Sondergaard BC, Schultz N, Madsen SH, et al. MAPKs are essential upstream signaling pathways in proteolytic cartilage degradation--divergence in pathways leading to aggrecanase and MMP-mediated articular cartilage degradation. Osteoarthritis Cartilage. 2010; 18(3): 279-88.

25. Nakai K, Kawato T, Morita T, et al. Angiotensin II induces the production of MMP-3 and MMP-13 through the MAPK signaling pathways via the $\mathrm{AT}_{1}$ receptor in osteoblasts. Biochimie. 2013; 95(4): 922-33.

26. Vande Geest JP, Di Martino ES, Vorp DA. An analysis of the complete strain field within Flexercell membranes. J Biomech. 2004; 37(12): 1923-8.

27. Shimizu N, Yamaguchi M, Goseki T, et al. Inhibition of prostaglandin $E_{2}$ and interleukin 1- $\beta$ production by low-power laser irradiation in stretched human periodontal ligament cells. J Dent Res. 1995; 74(7): 1382-8.

28. Jacobs C, Grimm S, Ziebart T, et al. Osteogenic differentiation of periodontal fibroblasts is dependent on the strength of mechanical strain. Arch Oral Biol. 2013; 58(7): 896-904.

29. Fushiki R, Mayahara K, Ogawa M, et al. High-magnitude mechanical strain inhibits the differentiation of bone-forming rat calvarial progenitor cells. Connect Tissue Res. 2015; 56(4): 336-41.

30. Laemmli UK. Cleavage of structural proteins during the assembly of the head of bacteriophage T4. Nature. 1970; 227(5259): 680-5.

31. Yanagisawa M, Suzuki N, Mitsui N, et al. Effects of compressive force on the differentiation of pluripotent mesenchymal cells. Life Sci. 2007; 81(5): 405-12.

32. Kanno T, Takahashi T, Tsujisawa T, et al. Mechanical stress-mediated Runx2 activation is dependent on Ras/ERK1/2 MAPK signaling in osteoblasts. J Cell Biochem. 2007; 101(5): 1266-77.

33. Yan YX, Gong YW, Guo Y, et al. Mechanical strain regulates osteoblast proliferation through integrin-mediated ERK activation. PLoS One. 2012; 7(4): e35709.

34. Yang CM, Chien CS, Yao CC, et al. Mechanical strain induces collagenase-3 (MMP-13) expression in MC3T3-E1 osteoblastic cells. J Biol Chem. 2004; 279(21): 22158-65.

35. Mu C, Lv T, Wang $\mathrm{Z}$, et al. Mechanical stress stimulates the osteo/odontoblastic differentiation of human stem cells from apical papilla via ERK 1/2 and JNK MAPK pathways. Biomed Res Int. 2014; 2014: 494378.

36. Bandow K, Nishikawa $\mathrm{Y}$, Ohnishi $\mathrm{T}$, et al. Low-intensity pulsed ultrasound (LIPUS) induces RANKL, MCP-1, and MIP-1 $\beta$ expression in osteoblasts through the angiotensin II type 1 receptor. J Cell Physiol. 2007; 211(2): 392-8.

37. Jackson RA, Kumarasuriyar A, Nurcombe V, et al. Long-term loading inhibits ERK1/2 phosphorylation and increases FGFR3 expression in MC3T3-E1 osteoblast cells. J Cell Physiol. 2006; 209(3): 894-904.

38. Matsuda N, Morita N, Matsuda K, et al. Proliferation and differentiation of human osteoblastic cells associated with differential activation of MAP kinases in response to epidermal growth factor, hypoxia, and mechanical stress in vitro. Biochem Biophys Res Commun. 1998; 249(2): 350-4.

39. Zhong HM, Ding $\mathrm{OH}$, Chen WP, et al. Vorinostat, a HDAC inhibitor, showed anti-osteoarthritic activities through inhibition of iNOS and MMP expression, p38 and ERK phosphorylation and blocking NF-KB nuclear translocation. Int Immunopharmacol. 2013; 17(2): 329-35.

40. Chen $\mathrm{K}$, Zhang S, Ji Y, et al. Baicalein inhibits the invasion and metastatic capabilities of hepatocellular carcinoma cells via down-regulation of the ERK pathway. PLoS One. 2013; 8(9): e72927.

41. Xue P, Zhao Y, Liu Y, et al. A novel compound RY10-4 induces apoptosis and inhibits invasion via inhibiting STAT3 through ERK-, p38-dependent pathways in human lung adenocarcinoma A549 cells. Chem Biol Interact. 2014; 209: 25-34.

42. Tower GB, Coon CC, Benbow U, et al. Erk $1 / 2$ differentially regulates the expression from the $1 \mathrm{G} / 2 \mathrm{G}$ single nucleotide polymorphism in the MMP-1 promoter in melanoma cells. Biochim Biophys Acta. 2002; 1586(3): 265-74.

43. Li Y, Tang L, Duan Y, et al. Upregulation of MMP-13 and TIMP-1 expression in response to mechanical strain in MC3T3-E1 osteoblastic cells. BMC Res Notes. 2010; 3: 309

44. Harada K, Kawai S, Wen-an X, et al. Alterations in Deciduous dental pulp cells cultured with serum-free medium. J Hard Tissue Biol. 2015; 24(1): 17-22.

45. Sun HB, Yokota H. Reduction of cytokine-induced expression and activity of MMP-1 and MMP-13 by mechanical strain in MH7A rheumatoid synovial cells. Matrix Biol. 2002; 21(3): 263-70.

46. Maeda E, Shelton JC, Bader DL, et al. Differential regulation of gene expression in isolated tendon fascicles exposed to cyclic tensile strain in vitro. J Appl Physiol. 2009; 106(2): 506-12.

47. Jones ER, Jones GC, Legerlotz K, et al. Cyclical strain modulates metalloprotease and matrix gene expression in human tenocytes via activation of TGF $\beta$. Biochim Biophys Acta. 2013; 1833(12): 2596-607.

48. Tang L, Lin Z, Li YM. Effects of different magnitudes of mechanical strain on osteoblasts in vitro. Biochem Biophys Res Commun. 2006; 344(1): 122-8. 